\title{
Prevalência de anemias em gestantes do município de Campo Mourão - PR entre os períodos de 2005 a 2008
}

\section{Prevalence of anemia among pregnant women in the city of Campo Mourão - PR between the periods 2005 to 2008}

\author{
Soraya Cristina Medeiros Américo ${ }^{1}$; Fabiana Nabarro Ferraz ${ }^{2}$
}

\begin{abstract}
Resumo
As gestantes constituem um dos grupos mais vulneráveis a adquirir anemias (carenciais). Este trabalho teve como objetivo verificar a prevalência de anemias em gestantes atendidas em Unidades Básicas de Saúde em Campo Mourão, de 2005 a 2008. Como instrumento de coleta de dados, foram utilizados os prontuários das gestantes e as seguintes variáveis: idade, situação conjugal, escolaridade, dosagem de hemoglobina e medicamentos prescritos para anemia. A prevalência de anemias nas gestantes foi de $6,18 \%$ e a maioria $(70,07 \%)$ apresentava valores de hemoglobina entre 9,6 a 10,8 g/dL. A faixa etária mais acometida encontrava-se entre 18 a 26 anos $(55,12 \%)$ e, com relação ao grau de escolaridade, a maioria $(62,20 \%)$ possuía ensino fundamental e médio completo e cerca da metade das gestantes eram casadas. O medicamento antianêmico mais prescrito foram os sais de ferro $(88,18 \%)$. A suplementação oral de ferro é a intervenção padrão para prevenção e tratamento da anemia durante a gestação. Neste estudo a prevalência de gestantes anêmicas foi considerada segundo a OMS um leve problema de saúde pública, sendo a grande maioria classificada como anemia moderada. Os resultados obtidos em nosso estudo possuem grande relevância, principalmente como fonte de futuras comparações e monitoramento da saúde na população de gestantes de Campo Mourão.

Palavras-chave: Anemia. Gestantes. Prevalência. Saúde pública.
\end{abstract}

\begin{abstract}
Pregnant women are one of the most vulnerable groups to acquire anemia (deficiency). Based on it, this study aimed verifying the prevalence of anemia among pregnant women, who were taken cared in Basic Health Units in Campo Mourão, from 2005 to 2008. As a tool for data collection were used the medical registrations of pregnant women and the following variables: age, marital status, hemoglobin dosage, and prescribed drugs for anemia. The prevalence of anemia among pregnant women was $6.18 \%$, the majority $(70.07 \%$ ) had hemoglobin values between 9.6 to $10.8 \mathrm{~g} / \mathrm{dL}$, the most affected age group was between 18 and $26(55.12 \%)$, in relation to the level of education, the majority $(62.20 \%)$ had primary and secondary school complete and about half of the women were married, the most prescribed medication for anemia were the salts of iron (88.18\%). Oral supplementation of iron is the standard intervention for prevention and treatment of anemia during pregnancy. In this study, the prevalence of pregnant women with anemia was considered, according to the WHO, a mild public health problem, which most cases being classified as moderate anemia. The results obtained in our study are very important, especially as a source for future comparisons and monitoring the health of pregnant women in Campo Mourão.
\end{abstract}

Keywords: Anemia. Pregnant women. Prevalence. Public health.

1 Farmacêutica formada pela Faculdade Integrado de Campo Mourão - PR.

2 Docente da disciplina de Hematologia Clínica. Curso de Farmácia. Departamento de Análises Clínicas e Biomedicina. Universidade Estadual de Maringá - PR. Av. Colombo, 5790. CEP 87020-900. E-mail: fabiana_nabarro@hotmail.com 


\section{Introdução}

A anemia em gestantes é definida pela Organização Mundial de Saúde (OMS) como a condição na qual o conteúdo de hemoglobina no sangue está abaixo do normal, ou seja, inferior a $11,0 \mathrm{~g} / \mathrm{dL}$, independentemente do trimestre gestacional (WALTER et al., 1989; DEMAEYER et al., 1991; LOZOFF et al., 2000; SABOOJEE; PETTIFOR, 2001; WORLD HEALTH ORGANIZATION, 2001).

A anemia caracteriza-se pela diminuição do número das células que transportam o oxigênio (glóbulos vermelhos) e/ou pela diminuição da hemoglobina que existe no interior destas células. A anemia é um sintoma-sinal originado por vários distúrbios orgânicos subjacentes, não sendo, uma doença em si, mas uma conseqüência de alterações que comprometem o binômio hemácia - hemoglobina. De modo simplificado, pode-se dizer que em qualquer tipo de anemia existe déficit de hemoglobina e/ou de hemácias circulantes (ZAGO; FALCÃO; PASQUINI, 2004).

As anemias podem ser classificadas, segundo a etiologia, em anemias cuja produção de eritrócitos está alterada (anemias carenciais - deficiência de ferro, vitamina B12, folato), anemias devido ao aumento da destruição dos eritrócitos (anemias hemolíticas) ou anemias devido a perdas sanguíneas (hemorragias) (WORLD HEALTH ORGANIZATION, 2001).

As anemias carenciais, embora encontradas em todos os países, é mais prevalente em países em desenvolvimento do que nos industrializados (CENTER FOR DISEASES CONTROL AND PREVENTION, 2002; WORLD HEALTH ORGANIZATION, 2000/2001; PAN AMERICAN HEALTH ORGANIZATION, 2003). Dados apontam no Brasil uma prevalência de anemia de até $50 \%$ em crianças de seis meses a cinco anos, 15 a $30 \%$ em gestantes, $20 \%$ em mulheres em idade fértil e $20 \%$ em adolescentes
(BRASIL, 2003; PAN AMERICAN HEALTH ORGANIZATION, 2003).

As gestantes constituem um dos grupos mais vulneráveis em desenvolver anemias, principalmente as carenciais, isso devido ao grande requerimento de micronutrientes neste período. Se a ingestão dietética for insuficiente e os estoques de nutrientes da mãe estiverem baixos, o feto precisará recorrer às reservas pré-concepcionais para se suprir, ocorrendo comprometimento do binômio materno-fetal. De forma geral, a diminuição na ingestão de micronutrientes pode provocar alterações dos mecanismos maternos de adaptação à gravidez. Percebe-se que, durante a gestação, se houver uma boa nutrição, é provável que os bebês nasçam mais saudáveis, já que um bom estado nutricional materno proporciona um bom desenvolvimento intra-uterino (THIAPO et al., 2007).

A anemia na gestação é um problema de Saúde Pública, devido aos efeitos prejudiciais que ocasiona na saúde e na qualidade de vida da gestante e do feto. Estudos evidenciam que a anemia na gravidez relaciona-se com maior risco de prematuridade, baixo peso ao nascer, mortalidade perinatal e menor concentração de hemoglobina no recém nascido (SANTOS; CERQUEIRA, 2008).

A anemia pode ser classificada em relação ao seu nível de importância em saúde pública, segundo a OMS, em: normal quando a prevalência for $<4,9 \%$; leve quando a prevalência estiver entre $5 \%$ a $19,9 \%$; moderada quando a prevalência estiver entre $20 \%$ a $39,9 \%$ e grave quando a prevalência for $>40 \%$. (WORLD HEALTH ORGANIZATION, 2001)

Com as modificações fisiológicas, tornase difícil o reconhecimento de condições patológicas e assim, considera-se que o adequado diagnóstico de anemia na gestação exige, por vezes, extensa investigação laboratorial: dosagem de hemoglobina, VCM (Volume corpuscular 
médio), ferritina, ferro sérico, capacidade total de fixação de ferro no sangue periférico e até mesmo biópsia de medula óssea, além dos antecedentes anamnéticos de cada caso (REZENDE; COLOVSKY, 1998).

A gravidez é um período em que as mulheres sofrem várias mudanças, tendo que se adaptar ao aumento de peso e a readaptação do organismo às proteínas, açúcares, minerais e ferro. Assim, a partir do primeiro trimestre, o volume de sangue tende a aumentar, expandindo com maior rapidez no segundo trimestre e mais lentamente no final da gestação. Esse processo ocorre em desigualdade, pois o volume líquido (plasma) é três vezes maior que o dos elementos figurados do sangue (glóbulos vermelhos, glóbulos brancos e plaquetas) (LUND; DONOVAN, 1967; BRANCH, 1992; KAISER, 2002). No entanto, os eritrócitos devem permanecer normocíticos e normocrômicos, a menos que ocorra deficiência de ferro, os valores normais tendem a retornar aos valores pré-gravídicos no puerpério, em torno de 6 a 8 semanas após o parto (BERNARD et al., 2000).

Este trabalho teve como objetivo verificar a prevalência de anemias em gestantes atendidas em Unidades Básicas de Saúde no município de Campo Mourão - PR, durante os períodos de 2005 a 2008.

\section{Material e métodos}

Foi realizado um estudo descritivo transversal retrospectivo, com dados de gestantes atendidas em Unidades Básicas de Saúde e Associação Beneficente Hospitalar Santa Casa de Misericórdia no município de Campo Mourão, Paraná.

A rede de atenção básica de saúde de Campo Mourão é constituída por 11 Unidades Básicas de Saúde (Centro Social Urbano, Damferi, Jardim Alvorada, Jardim Modelo, Jardim Paulista, Jardim Urupês, Lar Paraná, Vila Guarujá, Cohapar e
Piquirivaí) e mais a Santa Casa. Delas, nove foram incluídas no estudo (Centro Social Urbano, Damferi, Jardim Alvorada, Jardim Modelo, Jardim Paulista, Jardim Urupês, Lar Paraná, Vila Guarujá) e Santa Casa, e duas foram excluídas, devido à falta dos prontuários de segmento de pré-natal das gestantes no período de 2005 a 2008.

A coleta de dados foi realizada nos anos de 2005 a 2008 e, como instrumento de coleta de dados foram utilizados os prontuários de segmento de pré-natal das gestantes, contendo as seguintes variáveis: idade, situação conjugal, escolaridade, dosagem de hemoglobina (foram consideradas anêmicas as gestantes que possuíam dosagens de hemoglobina inferior a $11 \mathrm{~g} / \mathrm{dL}$, segundo OMS) e medicamentos prescritos para anemia na gestação.

A pesquisa no decorrer do seu desenvolvimento foi realizada de acordo com as normas da Resolução CNS (Conselho Nacional de Saúde) 196/96, após o consentimento do responsável, o secretário da saúde de Campo Mourão e aprovação do Comitê de Ética em Pesquisas com Seres Humanos, da Faculdade Integrado de Campo Mourão (registro No 4409).

Os dados obtidos foram organizados utilizando o programa EpiInfo 3.3.2 e as freqüências de cada dado foram calculadas.

\section{Resultados}

Segundo os dados obtidos nesta pesquisa, existia no período de 2005 a 2008, nas nove Unidades Básicas de Saúde (UBS) estudadas (Centro Social Urbano, Damferi, Jardim Alvorada, Jardim Modelo, Jardim Paulista, Jardim Urupês, Lar Paraná, Vila Guarujá) e mais a Santa Casa um total de 2054 prontuários de segmento de pré-natal de gestantes. Desse total, 127 gestantes foram diagnosticadas com anemia, ou seja, com dosagem de hemoglobina inferior a $11 \mathrm{~g} / \mathrm{dL}$ (Tabela 1). 
A prevalência de anemias observada nas Damferi com 15,29\% (13/85), seguida pela UBSgestantes estudadas foi de 6,18\%, variando em Urupês com 8,52\% (38/446) e UBS-Centro Social cada UBS. Foram as mais prevalentes, a UBS- Urbano com 7,65\% (31/405) (Tabela 1).

Tabela 1. Prevalência de anemias em gestantes atendidas no pré-natal nas unidades básicas de saúde do Município de Campo Mourão - PR nos períodos de 2005 -2008.

\begin{tabular}{lccc}
\hline Unidade Básica de Saúde & $\begin{array}{c}\text { Número total } \\
\text { de gestantes }\end{array}$ & $\begin{array}{c}\text { Número de } \\
\text { gestantes anêmicas }\end{array}$ & Prevalência de anemia \\
\hline Centro Social Urbano & 405 & $\mathrm{~N}$ & $\%$ \\
Damferi & 85 & 31 & 7,65 \\
Jardim Alvorada & 221 & 13 & 15,29 \\
Jardim Modelo & 172 & 07 & 3,16 \\
Jardim Paulista & 325 & 08 & 4,65 \\
Jardim Tropical & 139 & 11 & 3,38 \\
Jardim Urupês & 446 & 02 & 1,43 \\
Lar Paraná & 42 & 38 & 8,52 \\
Santa Casa & 145 & 02 & 4,76 \\
Vila Guarujá & 74 & 10 & 6,89 \\
\hline
\end{tabular}

A média de idade das gestantes anêmicas estudadas, calculada em anos, foi de 23,51 anos, com faixa de distribuição de 14 a 42 anos, sendo a faixa etária de 18 a 26 anos $(55,12 \%)$ a com maior freqüência de gestantes anêmicas.

Considerando o nível de escolaridade, foi observado que a maioria delas concluiu o ensino fundamental (32,28\%), seguido pelo ensino médio completo $(29,92 \%)$ e ensino fundamental incompleto $(24,40 \%)$. Com relação ao estado civil, cerca da metade das gestantes relataram serem casadas, seguido pelo estado civil solteira $(43,31 \%)$ (Tabela 2). 
Tabela 2. Perfil das gestantes anêmicas atendidas durante o pré-natal nas unidades básicas de saúde do município de Campo Mourão - PR nos períodos de 2005-2008.

\begin{tabular}{|c|c|c|c|c|c|c|c|c|}
\hline \multirow[t]{2}{*}{ Idade } & \multirow[b]{2}{*}{$\mathbf{N}$} & \multirow[b]{2}{*}{$\%$} & \multicolumn{2}{|l|}{ Escolaridade } & \multirow[b]{2}{*}{$\%$} & \multirow[t]{2}{*}{ Estado Civil } & \multirow[b]{2}{*}{$\mathbf{N}$} & \multirow[b]{2}{*}{$\%$} \\
\hline & & & & $\mathbf{N}$ & & & & \\
\hline$\overline{14+18}$ & 10 & 7,88 & Ensino Fund. & & & Casada & 60 & 47,24 \\
\hline $18 \vdash 22$ & 40 & 31,49 & Incompleto & 31 & 24,40 & & & \\
\hline $22+26$ & 30 & 23,63 & Ensino Fundamental & 41 & 32,38 & Separada & 1 & 0,78 \\
\hline $26-28$ & 15 & 11,82 & Ensino Médio & & & & & \\
\hline & & & Incompleto & 11 & 8,66 & Solteira & 55 & 43,31 \\
\hline $28+32$ & 19 & 14,96 & Ensino Médio & 38 & 29,92 & & & \\
\hline $32+36$ & 9 & 7,08 & Ensino Superior & & & Solteira com união & 10 & 7,89 \\
\hline $36+40$ & 3 & 2,36 & Incompleto & 3 & 2,36 & & & \\
\hline $40-44$ & 1 & 0,78 & Ensino Superior & 3 & 2,36 & Viúva & 1 & 0,78 \\
\hline
\end{tabular}

A Tabela 3 demonstra que a concentração de hemoglobina da maioria $(70,07 \%)$ das gestantes anêmicas, encontrava-se na faixa de 9,6 a 10,8 mg/dL. Em relação aos medicamentos antianêmicos prescritos, observou-se que a prescrição variou de acordo com a UBS, ou seja, com ginecologista e obstetra que nela trabalhava; os medicamentos mais utilizados para reposição de nutrientes (ferro, vitamina B12 e ácido fólico) foram, os sais de ferro $(88,18 \%)$, seguido pelo complexo B $(17,32 \%)$ e Combirom $(5,51 \%)$, e sais de ferro foram o principal meio de prevenção e reversão da anemia durante o período de gestação. 
Tabela 3. Dosagem de hemoglobina e medicamentos antianêmicos prescritos para gestantes anêmicas atendidas no pré-natal nas unidades básicas de saúde do Município de Campo Mourão - PR, nos períodos de $2005-2008$

Hemoglobina (g/dL)

\begin{tabular}{llllll} 
& $\mathbf{N}$ & $\mathbf{\%}$ & & $\mathbf{N}$ & $\mathbf{\%}$ \\
\hline $6,0+6,6$ & 1 & 0,78 & Combirom & 7 & 5,51 \\
$6,6+7,2$ & - & - & & & \\
$7,2+7,8$ & - & - & Complexo B & 22 & 17,32 \\
$7,8+8,4$ & - & - & & 1 & \\
$8,4+9,0$ & 3 & 2,38 & Neutrofer & & 0,78 \\
$9,0+9,6$ & 10 & 7,88 & & 1 & 0,78 \\
9,6 - 10,2 & 25 & 19,68 & Noripurum & & \\
$10,2+10,8$ & 64 & 50,39 & & & 88,18 \\
$10,8+11,2$ & 24 & 18,89 & Sulfato ferroso & 112 & \\
\hline
\end{tabular}

\section{Discussão}

Neste estudo, foi obtida uma prevalência de $6,18 \%$ (127/2050) de gestantes diagnosticadas com anemia, no município de Campo Mourão, no período de 2005 a 2008. A prevalência encontrada neste estudo foi inferior ao índice considerado de importância alarmante pela OMS (valor igual ou superior a $40 \%$ ) (WORLD HEALTH ORGANIZATION, 2000/2001), porém ainda pode ser considerado um leve problema de saúde pública, com a prevalência de gestantes anêmicas excedendo a 5\% (UNITED NATIONS CHILDREN'S FUND, 2001).

Em relação à faixa etária, foi observado que a maior prevalência de anemia em mulheres gestantes encontrava-se entre 18 a 26 anos (55,12\%), condizendo com estudos que afirmam que crianças, adolescentes, mulheres em idade reprodutiva e gestantes são considerados os principais grupos de risco para o desenvolvimento de anemias carênciais (KRAEMER; ZIMMERMANN, 2007).

A anemia é um problema global de saúde pública que afeta tanto os países desenvolvidos como os em desenvolvimento, com conseqüências graves para a saúde humana. Ela ocorre em todas as fases do ciclo de vida, mas é mais prevalente em mulheres grávidas e crianças pequenas (WORLD HEALTH ORGANIZATION, 2001).

No Brasil, a deficiência de ferro tem sido reconhecida como importante causa de anemia há mais de duas décadas. Segundo as estimativas da OMS, a anemia afeta $30 \%$ da população mundial, e pelo menos metade desta prevalência global pode ser atribuída à deficiência de ferro (CYRIL; HYANCINTH, 2007).

As gestantes são um dos grupos mais vulneráveis à anemia ferropriva, isso pelo aumento das necessidades desse mineral, não só devido ao crescimento fetal e placentário, como também ao aumento da volemia decorrente da gravidez (MARTINS et al., 1987). A anemia na gravidez, especialmente por carência de ferro, pode ocasionar: o aumento da mortalidade materna, mortalidade perinatal, prematuridade do 
recém nascido, baixo peso ao nascer e morbidade do feto (SCHOLL; REILLY, 2000; RASMUSSEN, 2001; WORLD HEALTH ORGANIZATION, 2001).

Estas intercorrências ocasionadas pela anemia durante a gestação demonstram a importância do acompanhamento médico do pré-natal, desde o início da gravidez como modo de prevenção, controle e tratamento, garantindo uma gravidez segura para a mãe e à criança em desenvolvimento.

Com relação ao grau de escolaridade a maioria das gestantes anêmicas possuem ensino fundamental e médio completo, totalizando uma prevalência de $62,20 \%$. Segundo Santos e Cerqueira (2008) a maior ocorrência de anemia bem como de outras deficiências nutricionais decorrem do nível educacional e cultural dos indivíduos. A baixa escolaridade também se reflete no reduzido número de oportunidades de emprego e de renda, com maior dificuldade de acesso aos alimentos e serviços de saúde (OSÓRIOS, 2002).

Para o diagnóstico de anemia em gestantes atendidas nas UBS de Campo Mourão, o critério médico utilizado é apenas o exame de hemograma, que é obrigatório no início do pré-natal, e no qual é analisada a dosagem de hemoglobina. Este critério médico de diagnóstico de anemia corresponde ao exame laboratorial preconizado pelo Ministério da Saúde para anemia gestacional, considerando sua praticidade e baixo custo (BRASIL, 2003). Mas alguns autores ainda contestam que o diagnóstico de anemia ferropriva deveria ser realizado após a combinação do resultado de hemoglobina com o de outra medida bioquímica, como a ferritina sérica ou receptor de transferrina, pois assim, com a utilização de mais de um indicador, aumenta-se consideravelmente a especificidade do diagnóstico (COOK; SKIKNE; BAYNES, 1992; WINTROBE; LUKERS; LEE, 1993; SCHWARTZ; THURNAU, 1995; WORLD HEALTH ORGANIZATION, 2000/2001; REVEIZ ; GYTE; CUERVO, 2007).

Neste estudo, observou-se que a maioria $(70,07 \%)$ das gestantes anêmicas apresentava anemia moderada, com valores entre 9,6 a 10,8 g/dL, de acordo com as definições de morbidade materna (7,0 g/dL a 10,0 g/dL) (CENTER FOR DISEASES CONTROL AND PREVENTION, 1989; WORLD HEALTH ORGANIZATION, 2000/2001).

As necessidades de ferro variam, acentuadamente, a cada trimestre gestacional. Os requerimentos não estão alterados no primeiro trimestre, devido à ausência do ciclo menstrual, apesar da vasodilatação generalizada e do aumento no volume plasmático circulante. A partir do segundo trimestre, esses requerimentos começam a se elevar em decorrência do aumento das necessidades de oxigênio para mãe e feto, perdurando até o final da gestação, e é necessário manter os níveis adequados de hemoglobina para garantir a saúde materno-fetal. Níveis adequados de hemoglobina são necessários durante a gestação para que o feto possa se desenvolver-se adequadamente. Caso contrário, o recém-nascido terá mais chance de desenvolver baixo peso, prematuridade ao nascer e aumento de morbidade (BOTHWELL, 2000; SHOBEIRIA; BEGUMB; NAZARIC, 2006).

Neste estudo o medicamento antianêmico mais prescrito pelos médicos das UBS de Campo Mourão, foram os sais de ferro com uma prevalência $88,18 \%$, porém algumas gestantes utilizavam mais que um medicamento antianêmico. Segundo Bashiri et al. (2003), a suplementação oral de ferro é considerada a intervenção padrão tanto para a prevenção como no tratamento da anemia durante a gestação.

Faz-se necessária a reposição de ferro durante a gestação com o objetivo de satisfazer o aumento dos requerimentos de ferro durante os dois últimos trimestres gestacionais, que são de extrema importância para o armazenamento deste mineral nos tecidos da criança em desenvolvimento para suprir suas necessidades logo após o nascimento (BOTHWELL, 2000; SHOBEIRIA; BEGUMB; NAZARIC, 2006).

O uso de medicamentos para a reposição de ferro durante a gestação deve-se à dificuldade de atendimento das necessidades diárias desse mineral apenas pela ingestão dietética, principalmente em 
países em desenvolvimento, onde o padrão alimentar geralmente apresenta baixa disponibilidade deste mineral (BOTHWELL, 2000).

A estratégia utilizada pelo serviço de saúde para sanar a deficiência de ferro é a suplementação medicamentosa com sais de ferro, devido ao seu baixo custo e boa absorção (EKSTRÖM et al 1996; SIMMONS et al 1993).

\section{Conclusão}

As anemias são consideradas um problema de saúde pública, tanto no Brasil como no mundo, afetando principalmente crianças, gestantes e mulheres em idade fértil, por isso se faz necessário estudos com o objetivo de verificar a prevalência, gravidade e a classe mais acometida para assim colocar em prática medidas corretivas e preventivas.

Neste estudo, a prevalência de gestantes anêmicas encontradas foi considerada segundo a OMS um leve problema de saúde pública, sendo a grande maioria classificada como anemia moderada. Esses dados demonstram a importância do seguimento prénatal e da realização do hemograma, com o intuito de diagnóstico de anemia, sendo a concentração da hemoglobina um dos parâmetros laboratoriais mais utilizado pelo clínico para o diagnóstico.

A efetividade da assistência pré-natal é determinante para se obter uma gestação segura e tranqüila e, entre outras questões, é imprescindível esclarecer para as gestantes a importância de uma alimentação equilibrada e rica em nutrientes, com o intuito de evitar o desenvolvimento das anemias carenciais. Cabe enfatizar, se necessário, o comprometimento da gestante em iniciar e terminar o tratamento com suplementação medicamentosa (sulfato ferroso, vitamina B12 e ácido fólico) de acordo com a prescrição médica, com o objetivo de se obter resultados satisfatórios desta terapia.

Os resultados obtidos no presente estudo possuem grande relevância, principalmente como fonte de futuras comparações e monitoramento da saúde na população de gestantes de Campo Mourão.

\section{Referências}

BASHIRI, A.; BURSTEIN, E.; SHEINER, E.; MAZOR, M. Anemia during pregnancy and treatment with intravenous iron: review of the literature. European Journal of Obstetrics and Reproductive Biology, Amsterdam, v. 110, n. 1, p. 2-7, 2003.

BERNARD, J.; LÉVY, J. P.; VARET, B.; CLAUVEL, J. P.; RAIN, J. D.; SULTAN, Y. Hematologia. 9. ed. Rio de Janeiro: Medsi, 2000.

BOTHWELL, T. H. Iron requirements in pregnancy and strategies to meet them. American Journal of Clinical Nutrition, New York, v. 72, p. S257-S64, 2000.

BRANCH, D. W. Physiologic adaptations of pregnancy. Journal of the American Reproductive Immunology, New York, v. 28, n. 102, p. 3-4, 1992.

BRASIL. Ministério da saúde. Política nacional de alimentação e nutrição. 2. ed. Brasília: Ministério da Saúde, 2003.

CENTER FOR DISEASE CONTROL AND PREVENTION. Current trends CDC criteria for anemia in children and childbearing-age women. Morbidity and Mortality Weekly Report, Atlanta, v. 38, p. 400-404, 1989.

CENTER FOR DISEASE CONTROL AND PREVENTION. Recomemendation to prevent and control fron deficiency - United States, 1999-2000. Morbidity and Mortality Weekly Report, Atlanta, v. 51, p. 897-899, 2002.

COOK, J. D.; SKIKNE, B. S.; BAYNES, R. D. Screening strategies for nutritional iron deficiency. In: FOMON, S. J.; ZLOTTKIN, S. (Ed.). Nutritional anemias. New York: Raven; 1992. p. 159-168. (Nestlé Nutrition Workshop Series, 30).

CYRIL, C. D.; HYANCINTH, E. O. The prevalence of anemia among pregnant women at booking in Enugu, South Eastern Nigéria. Medscape General Medicine, New York, v. 9, n. 3, p. 11, 2007.

DEMAEYER, E. M.; DALLMAN, P.; GURNEY, J.; HALLBERG, L.; SOOD, S. G. Preventing and controlling iron deficiency anaemia through primary health care: a guide for health administrators and programme managers. Geneva: World Health Organization, 1991. v. 58.

EKSTRÖM, E. C. M.; KAVISHE, F. P.; HABICHT, J. P.; FRONGILLO JUNIOR, E. A.; RASMUSSEN, K. M.; HEMED, L. Adherence to iron supplementation during pregnancy in Tanzania: determinants and hematologic consequences. American Journal of Clinical Nutrition, 
New York, v. 64, p. 368-374, 1996.

KAISER, L. L. Nutrition and lifestyle for a healthy pregnancy outcome. Journal of the American Dietetic Association, Chicago, v. 102, p. 1470-1490, 2002.

KRAEMER, K.; ZIMMERMANN, M. Nutritional anaemia. Basel: Sight and Life; 2007.

LOZOFF, B.; JIMENEZ, E.; HAGEN, J.; MOLLEN, E.; WOLF, A. W. Poorer Behavioral and Developmental Outcome More Than 10 Years After Treatment for Iron Deficiency in Infancy. Pediatrics, Springfield, v. 105, p. $1-4,2000$.

LUND, C. J.; DONOVAN, J. C. Blood volume during pregnancy: significance of plasma and red cell volumes. Journal of the American Gynecology Obstetrics, St. Louis, v. 98, n. 3, p. 394-403, 1967.

MARTINS, I. S.; ALVARENGA, A. T.; SZARFARC, S. C.; LIMA, F. D. As determinações biológica e social da doença: um estudo de anemia ferropriva. Revista de Saúde Pública, São Paulo, v. 21, n. 2, p. 73-89, 1987.

OSÓRIOS, M. M. Fatores determinantes da anemia em crianças. Jornal de Pediatria, Rio de Janeiro, v. 78, p. 269$278,2002$.

PAN AMERICAN HEALTH ORGANIZATION - OPAS. World Health Organization. Gender, health and evelopment in the America. 2003. Disponível em: <http://www.paho. org/english/ad/ge/genderbrochure05.pdf $>$. Acesso em: 15 Set. 2009.

RASMUSSEN, K. M. Iron deficiency anemia: reexamining the nature and magnitude of the public health problem: is there a causal relationship between iron deficiency or irondeficiency anemia and weight at birth, length of gestation and perinatal mortality? Journal of Nutrition, Philadelphia, v. 131, p. 590-603, 2001.

REVEIZ, L.; GYTE, G. M. L.; CUERVO, L. G. Treatments for iron-deficiency anaemia in pregnancy. Cochrane Database of Systematic Reviews, n. 2, 2007. Disponível em: $\quad<$ http://apps.who.int/rhl/reviews/CD003094.pdf $>$. Acesso em: 15 set. 2009.

REZENDE, J.; COLOVSKY, S. Repercussões da gestação sobre o organismo-modificações sistêmicas. In: REZENDE, J. Obstetrícia. 8. ed. Rio de Janeiro: Guanabara Koogan, 1998. p. 135.

SABOOJEE, H.; PETTIFOR, J. M. Iron deficiency and impaired child development. British Medical Journal, London, v. 323, p. 1377-1378, 2001.

SANTOS, P. N. P.; CERQUEIRA, E. M. M. Prevalência de anemia nas gestantes atendidas em unidades de Saúde em Feira de Santana, Bahia, entre Out. 2005 e Mar.2006. Revista Brasileira de Análises Clínicas, Rio de Janeiro, v.
40, n. 3, p. 219-223, 2008.

SCHOLL, T. O.; REILLY, T. Anemia, iron and pregnancy outcome. Journal of Nutrition, Philadelphia, v. 130, n. 2, p. 443, 2000.

SCHWARTZ, W. J.; THURNAU, G. R. Iron deficiency anemia in pregnancy. Clinical Obstetrics and Gynecology, Philadelphia, v. 38, n. 3, p. 443-454, 1995.

SHOBEIRIA, F.; BEGUMB, K.; NAZARIC, M. A prospective study of maternal hemoglobin status of Indian women during pregnancy and pregnancy outcome. Nutrition Research, Tarrytown, v. 26, n. 5, p. 209-213, 2006.

SIMMONS, W. K.; COOK, J. D.; BINGHAM, K. C.; THOMAS, M.; JACKSON, J.; AHLUWALIA, N.; KAHN, S. G.; PATTERSON, A. W. Evaluation of gastric delivery system for iron supplementation in pregnancy. American Journal of Clinical Nutrition, New York, v. 58, p. 622-626, 1993.

THIAPO, A. P.; LIBERA, B. D.; ACCIOLY, E; SOUZA, L. B., SAUNDERS, C., RAMALHO, A. Vitamina A, ferro e zinco na gestação e lactação. Revista Brasileira de Nutrição Clínica, São Paulo, v. 22, n. 2, p. 155-161, 2007.

UNITED NATIONS CHILDREN'S FUND/United Nations University/World Health Organization. Iron deficiency anemia: assessment, prevention, and control. Geneva: World Health Organization; 2001.

WALTER, T.; DE ANDRACA, I.; CHADUD, P.; PERALES, C. G. Iron deficiency anemia: adverse effects on infant psychomotor development. Pediatrics, Springfield, v. 84, p. 7-17, 1989.

WINTROBE, M. M.; LUKERS, J. N.; LEE, G. R. The approach to the patient with anemia. In: LEE, G. R.; BITHELL, T. C.; FOERSTER, J.; ATHENS, J. W.; LUKENS, J. N. Wintrobe's Clinical hematology. 9. ed. London: Lea \& Febiger, 1993. p. 715-744.

WORLD HEALTH ORGANIZATION - WHO. Iron deficiency anaemia: assessment, prevention, and control: a guide for programme managers. Geneva: WHO, 2000/2001.

ZAGO, M. A.; FALCÃO, R. P.; PASQUINI, R. Hematologia: fundamentos e prática. São Paulo: Atheneu, 2004.

Recebido em 7 de dezembro de 2009 - Received on December 7, 2009

Aceito em 27 de outubro de 2010 - Accepted on October 27, 2010 
